\title{
Influence of Age and Sex on the Blood Biochemical Constituent Values of Broiler Breeders During the Egg-Laying Stage
}

Marcelo Sebastião Rezende

Universidade Federal de Uberlandia

BELCHIOLINA BEATRIZ FONSECA ( $\sim$ biafonseca@ufu.br)

Universidade Federal de Uberlandia - Campus Umuarama https://orcid.org/0000-0001-8485-078X

Paula Fernanda Sousa Braga

Universidade Federal de Uberlandia

\section{Ednaldo Carvalho Guimarães}

Universidade Federal de Uberlandia

Antonio Vicente Mundim

Universidade Federal de Uberlandia

\section{Research Article}

Keywords: protein, metabolites, minerals, liver enzymes, skeletal muscle enzymes, blood.

Posted Date: July 9th, 2021

DOl: https://doi.org/10.21203/rs.3.rs-656808/v1

License: (c) (1) This work is licensed under a Creative Commons Attribution 4.0 International License.

Read Full License

Version of Record: A version of this preprint was published at Tropical Animal Health and Production on November 11th, 2021. See the published version at https://doi.org/10.1007/s11250-021-02981-z. 


\section{Abstract}

This study aimed to evaluate the influence of age and sex on the blood biochemical constituents of broiler breeders during the egg production stage. The analysis was performed in an industrial broiler breeder farm, and blood samples were collected from males and females at five different ages. At most ages, females had higher values of total proteins, albumin, globulins, triglycerides, cholesterol, glucose, calcium, phosphorus, $\mathrm{Ca} / \mathrm{P}$ (calcium and phosphorus)ratio and gamma-glutamyltransferase (GGT). The values of uric acid, aspartate aminotransferase (AST), creatine kinase (CK), and alkaline phosphatase (PAL) were higher in males. The lowest protein values were found at 28 and 60 weeks old. The mean albumin values were significantly higher at 44 and 52 weeks old in males and females. A trend of increasing globulin values with increasing age up to 52 weeks old was observed. Although calcium and phosphorus did not vary according to age, the $\mathrm{Ca} / \mathrm{P}$ ratio was lowest at 36 weeks old. Comparing the means of both sexes, the AST and GGT values were significantly higher in 60-week-old birds. The highest serum levels of CK occurred at 28 and 52 weeks old. The physiological levels of serum biomarkers presented in this work are important for evaluating productivity performance, welfare, and disease indication in breeding flocks.

\section{Introduction}

The Brazilian poultry industry has been outstanding in recent years due to genetic advances in obtaining broilers with excellent weight gain, high meat yield and low feed conversion.

Chicken productivity characteristics, such as high performance for weight gain, are transmitted by their male and female progenitors. Thus, the rearing of progenitors should be conducted to prioritize their reproductive characteristics. For females, these characteristics should aim to maximize fertile egg production with low mortality, and for males, these characteristics should guarantee good fertility and result in satisfactory hatch rates and progeny quality.

The performance of breeders is closely related to their environment, reflecting their individual interactions with exogenous factors such as environment, food, management and social relationships. Knowledge of the best physiological responses to these complex interactions is important to pursue the best performance, as this is an indicator of animal welfare.

Veterinary clinical pathology has stood out as an excellent tool for disease diagnosis, especially those derived from infectious and metabolic origins. Several studies in the poultry area have investigated some blood biochemical components to identify lines with better productive performance. The use of alanine aminotransferase (AST) as a biochemical marker for the disease known as "fatty liver syndrome" and the abdominal fat correlation with very-low-density lipoprotein (VLDL) serum levels are examples of applications of clinical pathology for the selection of animals with better productive performance (Hermier 1997; So et al. 2009). 
Although useful, blood biochemical components as disease markers in poultry are still insufficiently explored. Specifically, the study of characteristics that indicate high-performance birds of different ages for both sexes during the production period has not been explored in the literature. This study aimed to determine the physiological variations in certain blood biochemical constituents in male and female broiler breeders at the egg-laying stage, which is between 28 and 60 weeks old, and the influence of sex.

\section{Material And Methods}

This study was performed in healthy broiler breeders of Cobb lineage at an industrial production plant in Uberlândia, Minas Gerais, Brazil. The birds were raised in negative pressure barns at approximately $78.8^{\circ} \mathrm{F}$, and they remained healthy during all production periods. The flocks' egg production followed the standards recommended by the lineage.

During the egg-laying stage, females consumed layer feed number 1 from 25-48 weeks of age and layer feed 2 after 49 weeks of age (Supplementary table). Males consumed growth feed until 27 weeks of age and rooster feed from 28 weeks of age until the end of production (Supplementary table).

Thirty Cobb birds were randomly selected, with 15 females and 15 males in each of the following age groups: $28,36,44,52$ and 60 weeks old. This resulted in a total of 150 birds. Six hours after feed intake, the birds were weighed, and then, $3 \mathrm{ml}$ of blood was collected from each bird through ulnar vein puncture using disposable needles and syringes. As this manuscript is about the standardization of physiological variations in certain blood biochemical constituents in male and female broiler breeders at the egg-laying stage, a control group was not necessary. All the collect process was approved by Ethics Committee on the Use of Animals at the Federal University of Uberlândia.

Blood samples were transferred to sterile tubes without anticoagulant (dry tubes) immediately after collection. On the same day, the samples were sent to the Clinical Laboratory of the Veterinary Hospital of the Federal University of Uberlândia in an isothermal box and centrifuged at $720 \mathrm{x} \mathrm{g}$ for 10 minutes. The sera were separated and stored in microtubes at refrigeration temperature ( 35.6 to $46.4^{\circ} \mathrm{F}$ ) for 24 hours until the biochemical analysis procedure.

Biochemical elements analysed in each serum sample were: total proteins (Biuret's method), albumin (green bromocresol method), globulins (calculated as the difference between the total protein value and albumin), uric acid (Trinder enzyme method), total cholesterol and triglycerides (Trinder enzyme method), alanine aminotransferase (ALT) and aspartate aminotransferase (AST) (UV-IFCC kinetic method), gamma-glutamyltransferase (GGT) (modified Szasz method), creatine kinase (CK) (UV kinetic method), alkaline phosphatase (PAL) (Bowers and modified McComb method), calcium (cresolphthalein complexone method) and phosphorus (modified Daly and Ertingshausen method). A previously calibrated Labmax Plenno ${ }^{\circledR}$ automatic analyser (Calibra $\mathrm{H}$ ) was used and calibrated with universal control serum (Qualitrol 1), and commercial Labtest Diagnostica ${ }^{\circledR}$ kits were also used. The glycaemic status of each bird in the five age groups was determined shortly after the blood sample collection using the commercial One Touch ${ }^{\circledR}$ Ultra ${ }^{\text {TM }}$ glucometer. 
The experimental model adopted was a completely randomized design in a factorial scheme composed of two sexes, five ages and 15 replicates, totalling 150 samples. The Kolmogorov-Smirnov test with $5 \%$ significance was applied for the normality test, and the data showed normality. A t-test was used to compare differences between the sexes, and ANOVA was used to compare ages. These tests verified if the analysed data were statistically similar, with 5\% significance. GraphPad Prism 5.1 software was used.

\section{Results}

In this study, males and females had a weight gain compatible with their lineage (Cobb-Vantress 2018), with males having significantly higher weight gain than females at all ages (Table 1). The total protein, albumin, globulin, triglyceride and cholesterol serum levels were higher in females than in males at most evaluated ages (Table 1). Regarding age, the lowest protein values in females and males were found at the beginning ( 28 weeks old) and the end ( 60 weeks old) of the egg-laying stage. The mean albumin values of males and females were significantly higher at 44 and 52 weeks old. For both females and males, a trend of increasing globulin values with increasing age up to 52 weeks old was observed. Males had higher uric acid levels than females, and the value of uric acid in males and females increased with age. Regarding the age groups, the mean serum triglyceride levels in females and males were similar at all studied ages (Table 1). Females had higher glucose values than males at all studied ages, but there was no difference among ages (Table 1). 
Table 1

Mean values and standard deviations of weight, protein blood concentrations, metabolites and nutrients in broiler breeder males and females during the egg-laying stage.

\section{PARAMETER SEX AGE (weeks)}

\begin{tabular}{|c|c|c|c|c|c|c|}
\hline & & 28 & 36 & 44 & 52 & 60 \\
\hline Weight & $\mathrm{F}$ & $\begin{array}{l}3426.67^{\mathrm{B}} \pm \\
132.98\end{array}$ & $\begin{array}{l}3728.33^{\mathrm{B}} \pm \\
97.88\end{array}$ & $\begin{array}{l}3931.45^{\mathrm{B}} \pm \\
127.08\end{array}$ & $\begin{array}{l}4001.33^{B} \pm \\
115.16\end{array}$ & $\begin{array}{l}4003.21^{\mathrm{B}} \pm \\
160.57^{-}\end{array}$ \\
\hline \multirow[t]{2}{*}{ (g) } & $M$ & $\begin{array}{l}3960.33^{A} \pm \\
115.21\end{array}$ & $\begin{array}{l}4383.00^{\mathrm{A}} \pm \\
9.12\end{array}$ & $\begin{array}{l}4626.28^{\mathrm{A}} \pm \\
115.12\end{array}$ & $\begin{array}{l}4644.67^{A} \pm \\
100.06\end{array}$ & $\begin{array}{l}4808.33^{A} \pm \\
166.33\end{array}$ \\
\hline & $\begin{array}{l}\mathrm{F} \\
\text { and } \\
\mathrm{M}\end{array}$ & $\begin{array}{l}3693.50^{c} \pm \\
297.66\end{array}$ & $\begin{array}{l}4055.67^{b} \pm \\
345.40\end{array}$ & $\begin{array}{l}4278.87^{\mathrm{ab}} \pm \\
372.90\end{array}$ & $\begin{array}{l}4323.00^{\mathrm{a}} \pm \\
343.91\end{array}$ & $\begin{array}{l}4405.33^{a} \pm \\
440.24\end{array}$ \\
\hline $\begin{array}{l}\text { Total } \\
\text { proteins }\end{array}$ & $\mathrm{F}$ & $5.01^{A} \pm 0.69$ & $5.38^{A} \pm 0.67$ & $5.66^{A} \pm 0.46$ & $6.01^{A} \pm 0.53$ & $5.08^{A} \pm 0.34$ \\
\hline \multirow[t]{2}{*}{$\left(\mathrm{g} / \mathrm{dL}^{-1}\right)$} & $M$ & $3.61^{B} \pm 0.31$ & $5.01^{A} \pm 0.68$ & $4.35^{\mathrm{B}} \pm 0.36$ & $5.08^{B} \pm 0.51$ & $4.57^{B} \pm 0.67$ \\
\hline & $\begin{array}{l}\mathrm{F} \\
\text { and } \\
\mathrm{M}\end{array}$ & $4.31^{c} \pm 0.89$ & $\begin{array}{l}5.19^{\mathrm{ab}} \pm \\
0.69\end{array}$ & $\begin{array}{l}5.08 \text { ab } \\
\pm 0.76\end{array}$ & $5.55^{\mathrm{a}} \pm 0.70$ & $4.83^{b c} \pm 0.57$ \\
\hline Albumin & $\mathrm{F}$ & $2.09^{A} \pm 0.25$ & $1.93^{\mathrm{A}} \pm 0.26$ & $2.37^{A} \pm 0.19$ & $2.23^{\mathrm{A}} \pm 0.18$ & $1.90^{\mathrm{A}} \pm 0.13$ \\
\hline \multirow[t]{2}{*}{$\left(\mathrm{g} / \mathrm{dL}^{-1}\right)$} & $M$ & $1.37^{\mathrm{B}} \pm 0.12$ & $1.50^{B} \pm 0.13$ & $1.73^{\mathrm{B}} \pm 0.12$ & $1.77^{\mathrm{B}} \pm 0.16$ & $1.54^{\mathrm{B}} \pm 0.23$ \\
\hline & $\begin{array}{l}\mathrm{F} \\
\text { and } \\
\mathrm{M}\end{array}$ & $\begin{array}{l}1.74^{b} \pm 0 \\
.41\end{array}$ & $1.71^{b} \pm 0.32$ & $2.08^{a} \pm 0.37$ & $2.00^{\mathrm{a}} \pm 0.28$ & $1.73^{b} \pm 0.26$ \\
\hline Globulins & $\mathrm{F}$ & $2.92^{A} \pm 0.56$ & $2.80^{\mathrm{B}} \pm 0.16$ & $3.30^{\mathrm{A}} \pm 0.41$ & $3.79^{\mathrm{A}} \pm 0.48$ & $3.17^{A} \pm 0.26$ \\
\hline \multirow[t]{2}{*}{$\left(g / d^{-1}\right)$} & $M$ & $2.34^{\mathrm{B}} \pm 0.52$ & $3.34^{A} \pm 0.47$ & $2.63^{\mathrm{B}} \pm 0.31$ & $3.30^{\mathrm{B}} \pm 0.56$ & $3.03^{\mathrm{A}} \pm 0.50$ \\
\hline & $\begin{array}{l}\mathrm{F} \\
\text { and } \\
\mathrm{M}\end{array}$ & $2.63^{c} \pm 0.57$ & $3.07^{b} \pm 0.45$ & $3.00^{b c} \pm 0.47$ & $3.55^{\mathrm{a}} \pm 0.57$ & $3.10^{b} \pm 0.39$ \\
\hline \multirow[t]{3}{*}{$\mathrm{A} / \mathrm{G}$ ratio } & $\mathrm{F}$ & $0.73^{\mathrm{A}} \pm 0.13$ & $0.69^{\mathrm{A}} \pm 0.11$ & $0.73^{\mathrm{A}} \pm 0.11$ & $0.60^{A} \pm 0.09$ & $0.60^{A} \pm 0.04$ \\
\hline & $M$ & $0.58^{\mathrm{B}} \pm 0.19$ & $0.46^{\mathrm{B}} \pm 0.07$ & $0.66^{A} \pm 0.08$ & $0.55^{\mathrm{A}} \pm 0.10$ & $0.51^{B} \pm 0.07$ \\
\hline & $\begin{array}{l}\mathrm{F} \\
\text { and } \\
\mathrm{M}\end{array}$ & $\begin{array}{l}0.66^{\mathrm{ab}} \pm \\
0.13\end{array}$ & $\begin{array}{l}0.57^{b c} \pm \\
0.17 t\end{array}$ & $0.70^{\mathrm{a}} \pm 0.11$ & $\begin{array}{l}0.57^{b c} \pm \\
0.10\end{array}$ & $0.56^{c} \pm 0.07$ \\
\hline Uric Acid & $\mathrm{F}$ & $5.65^{\mathrm{B}} \pm 0.96$ & $5.22^{\mathrm{B}} \pm 0.91$ & $7.26^{\mathrm{B}} \pm 1.41$ & $6.44^{\mathrm{B}} \pm 1.32$ & $7.58^{\mathrm{B}} \pm 1.85$ \\
\hline$\left(\mathrm{mg} / \mathrm{dL}^{-1}\right)$ & M & $7.94^{A} \pm 3.31$ & $7.51^{A} \pm 2.89$ & $\begin{array}{l}11.07^{\mathrm{A}} \pm \\
3.28\end{array}$ & $\begin{array}{l}14.13^{\mathrm{A}} \pm \\
3.36\end{array}$ & $\begin{array}{l}11.79^{\mathrm{A}} \pm \\
2.84\end{array}$ \\
\hline
\end{tabular}




\section{PARAMETER SEX AGE (weeks)}

\begin{tabular}{|c|c|c|c|c|c|c|}
\hline & $\begin{array}{l}\mathrm{F} \\
\text { and } \\
\mathrm{M}\end{array}$ & $6.79^{b} \pm 2.66$ & $6.36^{b} \pm 2.34$ & $8.69^{a b} \pm 2.75$ & $\begin{array}{l}10.29^{a} \pm \\
4.64\end{array}$ & $9.61^{a} \pm 3.12$ \\
\hline Triglycerides & $\mathrm{F}$ & $\begin{array}{l}1120.07^{\mathrm{A}} \pm \\
205.97\end{array}$ & $\begin{array}{l}1589.57^{\mathrm{A}} \pm \\
246.10\end{array}$ & $\begin{array}{l}1556.40^{\mathrm{A}} \pm \\
131.70\end{array}$ & $\begin{array}{l}1538.60^{\mathrm{A}} \pm \\
66.66\end{array}$ & $\begin{array}{l}1380.33^{A} \pm \\
215.36\end{array}$ \\
\hline \multirow[t]{2}{*}{$\left(\mathrm{mg} / \mathrm{dL}^{-1}\right)$} & $M$ & $\begin{array}{l}77.07^{\mathrm{B}} \pm \\
18.45\end{array}$ & $\begin{array}{l}102.86^{\mathrm{B}} \pm \\
48.84\end{array}$ & $\begin{array}{l}94.88^{B} \pm \\
24.83\end{array}$ & $\begin{array}{l}92.33^{\mathrm{B}} \pm \\
17.45\end{array}$ & $\begin{array}{l}97.25^{\mathrm{B}} \pm \\
21.62\end{array}$ \\
\hline & $\begin{array}{l}\mathrm{F} \\
\text { and } \\
\mathrm{M}\end{array}$ & $\begin{array}{l}597.57^{\mathrm{a}} \pm \\
549.53\end{array}$ & $\begin{array}{l}846.21^{\mathrm{a}} \pm \\
776.43\end{array}$ & $\begin{array}{l}1048.04^{\mathrm{a}} \pm \\
747.14\end{array}$ & $\begin{array}{l}815.47^{\mathrm{a}} \pm \\
737.05\end{array}$ & $\begin{array}{l}810.07^{a} \pm \\
668.80\end{array}$ \\
\hline Cholesterol & $\mathrm{F}$ & $\begin{array}{l}155.13 \mathrm{~A} \pm \\
56.12\end{array}$ & $\begin{array}{l}170.29 \mathrm{~A} \pm \\
36.97\end{array}$ & $\begin{array}{l}187.60 \mathrm{~A} \pm \\
40.12\end{array}$ & $\begin{array}{l}182.47 \mathrm{~A} \pm \\
37.03\end{array}$ & $\begin{array}{l}145.67 \mathrm{~A} \pm \\
35.66\end{array}$ \\
\hline \multirow[t]{2}{*}{$(\mathrm{mg} / \mathrm{dL}-1)$} & M & $\begin{array}{l}94.07 \mathrm{~B} \pm \\
12.74\end{array}$ & $\begin{array}{l}123.00 \mathrm{~B} \pm \\
17.07\end{array}$ & $\begin{array}{l}110.00 \mathrm{~B} \pm \\
13.01\end{array}$ & $\begin{array}{l}119.00 \mathrm{~B} \pm \\
21.56\end{array}$ & $\begin{array}{l}113.64 \mathrm{~B} \pm \\
23.85\end{array}$ \\
\hline & $\begin{array}{l}\mathrm{F} \\
\text { and } \\
\mathrm{M}\end{array}$ & $\begin{array}{l}124.60 \mathrm{~b} \pm \\
50.63\end{array}$ & $\begin{array}{l}146.64 a b \pm \\
36.35\end{array}$ & $\begin{array}{l}158.50 \mathrm{a} \pm \\
48.88\end{array}$ & $\begin{array}{l}150.73 a b \pm \\
43.91\end{array}$ & $\begin{array}{l}130.21 \mathrm{ab} \pm \\
33.86\end{array}$ \\
\hline Glucose & $\mathrm{F}$ & $\begin{array}{l}250.40^{\mathrm{A}} \pm \\
16.33\end{array}$ & $\begin{array}{l}248.60^{\mathrm{A}} \pm \\
28.16\end{array}$ & $\begin{array}{l}256.53^{\mathrm{A}} \pm \\
19.21\end{array}$ & $\begin{array}{l}238.80^{\mathrm{A}} \pm \\
16.11\end{array}$ & $\begin{array}{l}248.67^{\mathrm{A}} \pm \\
19.08\end{array}$ \\
\hline \multirow[t]{2}{*}{$\left(\mathrm{mg} / \mathrm{dL}^{-1}\right)$} & M & $\begin{array}{l}220.80^{B} \pm \\
19.00\end{array}$ & $\begin{array}{l}200.13^{\mathrm{B}} \pm \\
16.08\end{array}$ & $\begin{array}{l}209.27^{B} \pm \\
22.22\end{array}$ & $\begin{array}{l}214.93^{\mathrm{B}} \pm \\
18.78^{-}\end{array}$ & $\begin{array}{l}195.47^{B} \pm \\
16.72\end{array}$ \\
\hline & $\begin{array}{l}\mathrm{F} \\
\text { and } \\
\mathrm{M}\end{array}$ & $\begin{array}{l}235.60^{\mathrm{a}} \pm \\
23.02\end{array}$ & $\begin{array}{l}224.37^{a} \pm \\
33.40\end{array}$ & $\begin{array}{l}232.90^{\mathrm{a}} \pm \\
31.53\end{array}$ & $\begin{array}{l}226.87^{\mathrm{a}} \pm \\
21.05\end{array}$ & $\begin{array}{l}222.07^{\mathrm{a}} \pm \\
32.29\end{array}$ \\
\hline \multicolumn{7}{|c|}{$\begin{array}{l}\text { Means followed by different capital letters in the same column or lowercase in the same row for the } \\
\text { same parameter evaluated differ by the Tukey test }(p<0.05) \text {. }\end{array}$} \\
\hline
\end{tabular}

The serum concentrations of calcium and phosphorus and the $\mathrm{Ca} / \mathrm{P}$ ratio were significantly higher in females than in males (Table 2). Serum calcium and phosphorus concentrations in females and males did not vary according to age. Among the age groups, the $\mathrm{Ca} / \mathrm{P}$ ratio was lowest at 36 weeks old (Table 2). 
Table 2

Mean values and standard deviations for mineral and enzyme serum concentrations in broiler breeder males and females during the egg laying stage.

\section{PARAMETER SEX AGE (weeks)}

\begin{tabular}{|c|c|c|c|c|c|c|}
\hline & & 28 & 36 & 44 & 52 & 60 \\
\hline Calcium & $\mathrm{F}$ & $\begin{array}{l}31.20 \mathrm{~A} \pm \\
7.91\end{array}$ & $\begin{array}{l}27.94 \mathrm{~A} \pm \\
4.03\end{array}$ & $\begin{array}{l}33.12 \mathrm{~A} \pm \\
2.98\end{array}$ & $\begin{array}{l}31.96 \mathrm{~A} \pm \\
3.68\end{array}$ & $\begin{array}{l}26.24 \mathrm{~A} \pm \\
3.87\end{array}$ \\
\hline \multirow[t]{2}{*}{$(\mathrm{mg} / \mathrm{dL}-1)$} & $M$ & $\begin{array}{l}12.53 \mathrm{~B} \pm \\
1.43\end{array}$ & $\begin{array}{l}10.70 \mathrm{~B} \pm \\
1.31\end{array}$ & $\begin{array}{l}12.60 \mathrm{~B} \pm \\
0.85\end{array}$ & $\begin{array}{l}14.40 \mathrm{~B} \pm \\
0.73\end{array}$ & $\begin{array}{l}13.26 \mathrm{~B} \pm \\
5.95\end{array}$ \\
\hline & $\begin{array}{l}\mathrm{F} \\
\text { and } \\
\mathrm{M}\end{array}$ & $\begin{array}{l}21.87 a \pm \\
11.02\end{array}$ & $\begin{array}{l}19.32 \mathrm{a} \pm \\
9.22\end{array}$ & $\begin{array}{l}25.42 \mathrm{a} \pm \\
10.57\end{array}$ & $\begin{array}{l}23.19 \mathrm{a} \pm \\
9.29\end{array}$ & $\begin{array}{l}19.97 \mathrm{a} \pm \\
8.19\end{array}$ \\
\hline Phosphorus & $\mathrm{F}$ & $9.46 \mathrm{~A} \pm 3.18$ & $9.50 A \pm 1.75$ & $9.87 A \pm 1.68$ & $\begin{array}{l}10.47 \mathrm{~A} \pm \\
1.81\end{array}$ & $8.01 \mathrm{~A} \pm 1.64$ \\
\hline \multirow[t]{2}{*}{$(\mathrm{mg} / \mathrm{dL}-1)$} & $M$ & $4.88 \mathrm{~B} \pm 0.76$ & $5.91 \mathrm{~B} \pm 0.67$ & $4.16 \mathrm{~B} \pm 0.48$ & $4.95 B \pm 0.52$ & $4.48 \mathrm{~B} \pm 2.26$ \\
\hline & $\begin{array}{l}\mathrm{F} \\
\text { and } \\
\mathrm{M}\end{array}$ & $7.17 a \pm 3.25$ & $7.71 a \pm 2.23$ & $7.28 a \pm 3.13$ & $7.71 a \pm 3.10$ & $6.31 a \pm 2.62$ \\
\hline \multirow[t]{3}{*}{$\mathrm{Ca} / \mathrm{P}$ ratio } & $\mathrm{F}$ & $3.39 A \pm 0.58$ & $2.96 \mathrm{~A} \pm 0.25$ & $3.44 \mathrm{~A} \pm 0.60$ & $3.09 A \pm 0.34$ & $3.33 \mathrm{~A} \pm 0.61$ \\
\hline & M & $2.61 \mathrm{~B} \pm 0.41$ & $1.82 B \pm 0.24$ & $3.07 \mathrm{~A} \pm 0.45$ & $2.94 \mathrm{~A} \pm 0.29$ & $2.70 \mathrm{~B} \pm 0.53$ \\
\hline & $\begin{array}{l}\mathrm{F} \\
\text { and } \\
\mathrm{M}\end{array}$ & $3.00 a \pm 0.63$ & $2.39 b \pm 0.62$ & $3.30 a \pm 0.53$ & $3.01 a \pm 0.32$ & $3.19 a \pm 0.48$ \\
\hline ALT & $\mathrm{F}$ & $\begin{array}{l}10.46 \mathrm{~A} \pm \\
4.45\end{array}$ & $\begin{array}{l}10.73 \mathrm{~A} \pm \\
2.41\end{array}$ & $\begin{array}{l}11.23 \mathrm{~A} \pm \\
3.65\end{array}$ & $9.80 \mathrm{~A} \pm 4.11$ & $\begin{array}{l}11.27 \mathrm{~A} \pm \\
2.02\end{array}$ \\
\hline \multirow[t]{2}{*}{$(\mathrm{UI} / \mathrm{L}-1)$} & $M$ & $9.93 \mathrm{~A} \pm 1.16$ & $9.36 \mathrm{~A} \pm 1.01$ & $8.78 A \pm 2.73$ & $\begin{array}{l}10.60 \mathrm{~A} \pm \\
3.54\end{array}$ & $\begin{array}{l}13.00 \mathrm{~A} \pm \\
3.14\end{array}$ \\
\hline & $\begin{array}{l}\mathrm{F} \\
\text { and } \\
\mathrm{M}\end{array}$ & $\begin{array}{l}12.47 a \pm \\
11.85\end{array}$ & $9.96 a \pm 1.75$ & $\begin{array}{l}10.38 \mathrm{a} \pm \\
3.41\end{array}$ & $\begin{array}{l}10.20 \mathrm{a} \pm \\
3.79\end{array}$ & $\begin{array}{l}12.07 a \pm \\
2.70\end{array}$ \\
\hline AST & $\mathrm{F}$ & $\begin{array}{l}103.60 \mathrm{~B} \pm \\
32.07\end{array}$ & $\begin{array}{l}203.64 \mathrm{~B} \pm \\
22.50\end{array}$ & $\begin{array}{l}137.93 \mathrm{~A} \pm \\
34.43\end{array}$ & $\begin{array}{l}206.67 \mathrm{~A} \pm \\
70.20\end{array}$ & $\begin{array}{l}269.00 \mathrm{~A} \pm \\
27.96\end{array}$ \\
\hline \multirow[t]{2}{*}{$(\mathrm{UI} / \mathrm{L}-1)$} & $M$ & $\begin{array}{l}145.80 \mathrm{~A} \pm \\
35.50\end{array}$ & $\begin{array}{l}278.64 \mathrm{~A} \pm \\
19.65\end{array}$ & $\begin{array}{l}106.78 \mathrm{~A} \pm \\
31.53\end{array}$ & $\begin{array}{l}85.07 \mathrm{~B} \pm \\
35.82\end{array}$ & $\begin{array}{l}274.14 \mathrm{~A} \pm \\
23.63\end{array}$ \\
\hline & $\begin{array}{l}\mathrm{F} \\
\text { and } \\
\mathrm{M}\end{array}$ & $\begin{array}{l}124.70 c \pm \\
39.57\end{array}$ & $\begin{array}{l}241.14 b \pm \\
43.05\end{array}$ & $\begin{array}{l}126.25 c \pm \\
33.24\end{array}$ & $\begin{array}{l}145.87 \mathrm{c} \pm \\
82.60\end{array}$ & $\begin{array}{l}271.66 \mathrm{a} \pm \\
25.08\end{array}$ \\
\hline CK & $\mathrm{F}$ & $\begin{array}{l}3800.35 \mathrm{~B} \pm \\
1822.05\end{array}$ & $\begin{array}{l}5312.25 \mathrm{~B} \pm \\
1650.80\end{array}$ & $\begin{array}{l}5410.30 \mathrm{~A} \pm \\
1841.11\end{array}$ & $\begin{array}{l}7388.99 A \pm \\
4738.20\end{array}$ & $\begin{array}{l}4252.53 \mathrm{~A} \pm \\
1132.60\end{array}$ \\
\hline
\end{tabular}




\begin{tabular}{|c|c|c|c|c|c|c|}
\hline PARAMETER & SEX & AGE (weeks) & & & & \\
\hline \multirow[t]{2}{*}{$(\mathrm{UI} / \mathrm{L}-1)$} & M & $\begin{array}{l}6652.80 \mathrm{~A} \pm \\
3308.14\end{array}$ & $\begin{array}{l}8758.88 \mathrm{~A} \pm \\
2299.59\end{array}$ & $\begin{array}{l}6666.39 \mathrm{~A} \pm \\
3106.51\end{array}$ & $\begin{array}{l}10087.43 \mathrm{~A} \pm \\
4285.64\end{array}$ & $\begin{array}{l}6284.34 \mathrm{~A} \pm \\
3517.50\end{array}$ \\
\hline & $\begin{array}{l}\mathrm{F} \\
\text { and } \\
\mathrm{M}\end{array}$ & $\begin{array}{l}5177.39 \mathrm{~b} \pm \\
2934.76\end{array}$ & $\begin{array}{l}7035.56 a b \pm \\
2581.52\end{array}$ & $\begin{array}{l}6152.54 b \pm \\
2496.47\end{array}$ & $\begin{array}{l}8738.21 a \pm \\
4646.29\end{array}$ & $\begin{array}{l}5233.40 \mathrm{~b} \pm \\
2707.83\end{array}$ \\
\hline GGT & $\mathrm{F}$ & $\begin{array}{l}\text { 19.67A } \pm \\
3.31\end{array}$ & $\begin{array}{l}15.71 \mathrm{~A} \pm \\
3.29\end{array}$ & $\begin{array}{l}30.33 \mathrm{~A} \pm \\
1.59\end{array}$ & $\begin{array}{l}43.60 \mathrm{~A} \pm \\
25.23\end{array}$ & $\begin{array}{l}62.60 \mathrm{~A} \pm \\
37.22\end{array}$ \\
\hline \multirow[t]{2}{*}{$(\mathrm{UI} / \mathrm{L}-1)$} & M & $\begin{array}{l}20.40 \mathrm{~A} \pm \\
3.72\end{array}$ & $\begin{array}{l}17.07 \mathrm{~A} \pm \\
9.36\end{array}$ & $\begin{array}{l}34.22 \mathrm{~A} \pm \\
9.16\end{array}$ & $\begin{array}{l}24.07 \mathrm{~B} \pm \\
3.13\end{array}$ & $\begin{array}{l}30.64 \mathrm{~B} \pm \\
8.98\end{array}$ \\
\hline & $\begin{array}{l}\mathrm{F} \\
\text { and } \\
\mathrm{M}\end{array}$ & $\begin{array}{l}20.03 c d \pm \\
3.48\end{array}$ & $\begin{array}{l}16.39 d \pm \\
6.68\end{array}$ & $\begin{array}{l}31.79 b c \pm \\
5.59\end{array}$ & $\begin{array}{l}33.83 b \pm \\
20.27\end{array}$ & $\begin{array}{l}47.17 a \pm \\
31.13\end{array}$ \\
\hline PAL & $\mathrm{F}$ & $\begin{array}{l}\text { 1017.27A } \pm \\
602.74\end{array}$ & $\begin{array}{l}212.93 \mathrm{~B} \pm \\
78.32\end{array}$ & $\begin{array}{l}261.67 \mathrm{~B} \pm \\
81.86\end{array}$ & $\begin{array}{l}181.73 \mathrm{~B} \pm \\
38.69\end{array}$ & $\begin{array}{l}642.14 \mathrm{~B} \pm \\
338.11\end{array}$ \\
\hline \multirow[t]{2}{*}{$(\mathrm{UI} / \mathrm{L}-1)$} & $M$ & $\begin{array}{l}1040.87 \mathrm{~A} \pm \\
671.57\end{array}$ & $\begin{array}{l}937.29 \mathrm{~A} \pm \\
84.92\end{array}$ & $\begin{array}{l}1198.11 \mathrm{~A} \pm \\
1144.14\end{array}$ & $\begin{array}{l}551.13 \mathrm{~A} \pm \\
518.79\end{array}$ & $\begin{array}{l}989.57 \mathrm{~A} \pm \\
484.73\end{array}$ \\
\hline & $\begin{array}{l}\mathrm{F} \\
\text { and } \\
\mathrm{M}\end{array}$ & $\begin{array}{l}1029.07 a \pm \\
627.10\end{array}$ & $\begin{array}{l}575.11 b c \pm \\
376.16\end{array}$ & $\begin{array}{l}612.83 b c \pm \\
174.03\end{array}$ & $\begin{array}{l}366.43 c \pm \\
407.37\end{array}$ & $\begin{array}{l}815.86 a b \pm \\
432.20\end{array}$ \\
\hline \multicolumn{7}{|c|}{$\begin{array}{l}\text { Means followed by different capital letters in the same column or lowercase in the same row for the } \\
\text { same parameter evaluated differ by the Tukey test }(p<0.05) \text {. }\end{array}$} \\
\hline \multicolumn{7}{|c|}{$\mathrm{F}=$ Females $\mathrm{M}=$ Males } \\
\hline
\end{tabular}

For ALT, there were no significant differences between males and females in any the different age groups (Table 2). Higher values of AST and CK were observed in males than in females at 28 and 36 weeks old. Comparing the means of both sexes, enzyme values were significantly higher in 60-week-old birds (Table 2). CK had the highest serum levels at 28 and 52 weeks old in females and males (Table 2). There were higher serum concentrations of GGT in 52- and 60-week-old females at the end of the reproductive period, and the average increased for both the sexes in these age groups (Table 2). When comparing the serum levels of PAL in this study, the males had higher levels than females at most of the studied ages, except for 28-week-old birds, where males and females had the same levels at the beginning of the egglaying stage. Evaluating the means obtained from females and males, the highest value of PAL was found at 28 weeks old (Table 2).

\section{Discussion}

Biochemical markers in the blood of high-performance broiler breeders during the egg-laying stage can be an essential tool for future interpretation of these biological markers for performance or even disease in 
these animals. In this study, males and females had a weight gain compatible with their lineage (CobbVantress 2018), with males having significantly higher weight gain than females at all ages (Table 1). This was an expected result because the birds on this farm were carefully raised following standardized management and health methods.

Protein serum levels in females were higher than those in males at most of the evaluated ages, except for 36 weeks, in which the values were similar between sexes. The female's high protein demand for egg production (Penz and Jensen 1991; Capitelli and Crosta 2013) can explain this result. The peak of egg production at 36 weeks can explain the similarity of serum protein values between males and females at this time. During peak production, females need a large amount of protein to form eggs, which can reduce the amount of serum protein. Considering the total protein mean of females and males, the lowest values were found at the beginning ( 28 weeks old) and the end ( 60 weeks old) of the egg-laying stage. This event probably occurred because of lower egg production in these two stages.

Albumin accounts for $40-50 \%$ of the total plasma protein in birds, with regular levels ranging from 0.8 to $2.0 \mathrm{~g} / \mathrm{dL}$ (Schmidt et al. 2007). Serum values presented by females were higher than those obtained by males. The high protein level that the females demand during egg production and the lower percentage of protein and amino acid levels in the male feed (Supplementary table) also reflected the lower values found for male serum albumin.

At most of the evaluated ages, the serum globulin content was higher in females than in males. Globulins consist of alpha globulins, beta globulins and gammaglobulins. This group of globulins includes acute phase proteins (a-globulins and $\beta$-globulins) and immunoglobulins ( $\gamma$-globulins) (Melillo 2013). Alpha globulins are involved in acute response processes during trauma, inflammation or infection situations, whereas beta globulins still play an unknown clinical role (Melillo 2013). During the egg-laying stage, oestrogen-induced hyperproteinaemia occurs to complete egg formation because most of the yolk proteins are globulins and cause a marked increase in globulin fractions (Campbell and Dein 1984; Capitelli and Crosta 2013).

When evaluating females and males, a trend of increasing globulin values with increasing age up to 52 weeks old was observed. Studies in cattle also identified increased globulin serum levels with increasing age (Liberg 1977). Hasegawa et al. (2002) studied the serum protein biochemical profile in broiler breeders and found a value of $2.7 \mathrm{~g} / \mathrm{dL}$ for globulins in birds at 63 weeks old. This value is a near the value found by us.

Males had higher uric acid levels than females (Table 1). Several authors have seen that roosters need lower protein levels than females to improve reproductive performance (Hocking 1990; Silveira et al. 2014), with an intake need of approximately $12 \%$ (Hocking 1990). Thus, the feed intake in this study had higher protein content (14\%) than in other studies, and this may have resulted in higher nitrogen metabolite production in males. Another possible explanation for the lower level of uric acid in females may be related to the need for an increase in the amount of protein for egg formation, which results in less excretion of this metabolite. In this study, the uric acid was increased according to age in these birds, 
and the values observed in 52- and 60-week-old birds were higher than those seen in 28- and 36-week-old birds (Table 1). Increased excretion of uric acid in males and females in the final reproductive stage indicates that the same protein intake was associated with lower production.

Serum triglyceride and cholesterol levels were significantly higher in females than in males (Table 1). During egg laying, oestrogen increases hepatic lipid production, mainly triglycerides, to establish a reserve of energy for the embryo (Walzern 1996). A significant increase in cholesterol plasma concentrations occurs in females during the egg-laying stage due to vitellogenesis for egg yolk formation (Harr 2002). Glycaemia in avian species is 150 to $300 \%$ higher than that in mammals, considering the same body mass (Braun and Sweazea 2008). Females had higher values of glucose than males at all studied ages (Table 2), probably due to the energy requirement for egg production.

Serum calcium and phosphorus concentrations found in females were significantly higher than those in males (Table 2). These blood levels are dietary-dependent (Viñuela et al. 1991). For females, calcium plays an essential role in eggshell formation; it is induced by oestrogen (Harr 2002; Dunbar et al. 2005) and transported by linked proteins, such as vitellogenin and albumin (Capitelli and Crosta 2013). Phosphorus metabolism is closely related to that of calcium, especially regarding absorption at serum levels. Higher calcium concentrations in females require proportionally higher phosphorus levels to maintain homeostasis of these two electrolytes (Proszkowiec-Weglarz and Angel 2013). According to age, in the birds from this study, serum phosphorus levels did not vary between females and males. As a result, in this study, the $\mathrm{Ca} / \mathrm{P}$ ratio was higher in females than males. This was mainly due to calcium serum levels, which were proportionally higher in females (Table 2). Among the age groups, the values were mostly the same, mainly due to the low variation of levels in females.

During the highest egg production stage, one of the most crucial liver roles is yolk production, but this did not result in increased ALT levels in females compared to males at the different ages. Although ALT elevations are associated with hepatic or muscular lesions in most birds, even those with severe hepatic lesions do not show significant variations in serum concentration of this enzyme (Hochleithner 1994). Thus, ALT in birds may not be an important bioindicator for birds with liver problems.

AST is not considered a specific enzyme of the hepatic and muscular tissues; however, changes in this enzyme in the blood are primarily associated with disorders in these tissues. In this study, the AST values were higher in males than in females at 28 and 36 weeks of age, possibly due to more significant physical activity during the competition for females. Comparing the means of both sexes, enzyme values were significantly higher in 60-week-old birds (Table 2) and may indicate hepatic or muscular tissue alterations due to age.

Creatine kinase (CK) is a specific enzyme for skeletal muscle, and its increase in birds is mainly associated with muscle changes (Capitelli and Crosta 2013). Comparing the results between sexes, at 28 and 36 weeks old, the serum levels of CK were higher in males than in females (Table 2). Previous studies have shown that in addition to its utility as a marker of muscle damage, CK can also be used to quantify the energy required for the reproductive strategy of each sex, aiding the study of these strategies (Ramírez 
et al. 2010). There are increases in CK and AST in the reproductive period, which is associated with adrenaline release, increased exercise and stress (Stout et al. 2010). The elevations in the levels of AST and CK observed in the birds of this study are consistent with the studies of Stout et al. (2010). Males, especially in the early stages of the reproductive period, experience very intense physical activity while competing with each other to form the family nucleus within the breeding stock.

Comparing the female and male means, the highest serum levels of CK occurred at 28 and 52 weeks of age (Table 2). There was management with the birds at these ages. Males underwent movement, containment and individual evaluation to select them according to their body shape to keep the capable ones in the flock. For this process, females are also involved since they are in the same environment as males. This process involves intense muscle activity, which is reflected in the increase in CK blood values.

There was a higher serum GGT concentration in 52- and 60-week-old females, which affected the average between the sexes in these age groups (Table 2). The high serum activity of GGT occurs due to increased production and release of GGT caused by hepatobiliary changes (Meyer 1995). Interestingly, simultaneous increases in GGT and AST levels at these same ages corroborate the suspicion of liver origin changes, mainly due to fatty infiltration, which can cause hepatic injury.

In this study, the males presented higher PAL levels than females at most of the evaluated ages, except at 28 weeks old. This age is the beginning of the egg-laying stage, so males and females had the same levels of PAL. PAL is related to bone metabolism, mainly with osteoblastic activity (Rajman et al. 2006). Compared with females, males had higher serum PAL values. This could possibly be due to a more significant amount of bone isoenzymes because they presented a greater amount of bone tissue due to their more developed skeleton. In another study, a higher level of PAL in males was also found when compared to females at 73 weeks old (Rath et al. 1999). When comparing the means obtained from females and males according to age, the highest value was obtained at 28 weeks old; the higher levels were mainly presented by females. This event probably occurs due to oestrogenic action, which stimulates osteoblasts to deposit calcium in the bone marrow, constituting a calcium mobilization reserve source for eggshell formation (Farmer et al. 1983). At the end of the reproductive period, an elevation of PAL levels was observed in 60-week-old birds in both females and males. There was a strong correlation with increased levels presented by females. This is probably due to the decrease in calcium absorption that occurs in older birds as oestrogen levels decrease, and, with this, there is greater bone resorption.

\section{Conclusion}

In healthy male and female broiler breeders during the egg-laying stage, there was a physiological difference in total proteins, albumin, globulins, $A / G$ ratio, uric acid, triglycerides, cholesterol, glucose, calcium, phosphorus, $\mathrm{Ca} / \mathrm{P}$ ratio, AST, CK, GGT and PAL between the sexes within the same age group. The serum biochemical parameter ALT did not significantly differ between the sexes within the same age group or among the different ages. 
This study presents an important reference for the biochemical parameters of blood proteins, lipids, enzymes, calcium and phosphorus in broiler breeders. These data will be useful for other studies that aim to improve the use of biochemical markers to assess the physiological state of birds.

\section{Declarations}

\section{FUNDING}

Part of this study was financed by the Coordenação de Aperfeiçoamento de Pessoal de Nível Superior Brasil (CAPES) - Finance Code 001, FAPEMIG (Fundação de Amparo à Pesquisa do Estado de Minas Gerais) and CNPq (Conselho Nacional de Desenvolvimento Científico e Tecnológico).

\section{CONFLICTS OF INTERESTS}

The authors declare no conflicts of interests.

\section{AVAILABILITY OF DATA AND MATERIAL}

The datasets generated during and/or analysed during the current study are available from the corresponding author on reasonable request.

\section{CODE AVAILABILITY}

Not applicable.

AUTHORS' CONTRIBUTIONS - Rezende and Mundim planned the research. Rezende and Braga collected sample. Mundim performed laboratory analysis. Guimarães performed the statistical analysis and interpreted the data. Rezende, Mundim, Fonseca interpreted the date. Rezende and Fonseca wrote the manuscript. Fonseca, Mundim and Braga carried out the revision

All authors have read and agreed to the published version of the manuscript.

\section{COMMITTEE ON ETHICS AND BIOSAFETY}

All procedures performed in this study were in accordance with Law No. 11.794, of October 8, 2008 certificated by Protocol CEUA/UFU 004/16, approved on March 15, 2016, by the Ethics Committee on the Use of Animals at the Federal University of Uberlândia.

Consent to participate (include appropriate statements): Not applicable.

\section{References}

1. Braun EJ, Sweazea KL (2008) Glucose regulation in birds. Comp Biochem Physiol Part B Biochem Mol Biol 151:1-9. https://doi.org/10.1016/j.cbpb.2008.05.007 
2. Campbell TW, Dein JF (1984) Avian Hematology. Vet Clin North Am Small Anim Pract 14:223-248. https://doi.org/10.1016/S0195-5616(84)50031-X

3. Capitelli R, Crosta L (2013) Overview of Psittacine Blood Analysis and Comparative Retrospective Study of Clinical Diagnosis, Hematology and Blood Chemistry in Selected Psittacine Species. Vet Clin North Am Exot Anim Pract 16:71-120. https://doi.org/10.1016/j.cvex.2012.10.002

4. Cobb-Vantress (2018) Suplemento para manejo de matrizes

5. Dunbar MR, Gregg MA, Crawford JA, et al (2005) Normal hematologic and biochemical values for prelaying greater sage grouse (Centrocercus Urophasianus) and their influence on chick survival. $J$ Zoo Wildl Med 36:422-429. https://doi.org/10.1638/04-065.1

6. Farmer M, Roland DA, Brake J, Eckman MK (1983) Calcium Metabolism in Broiler Breeder Hens. Poult Sci 62:459-464. https://doi.org/10.3382/ps.0620459

7. Harr KE (2002) Clinical Chemistry of Companion Avian Species: A Review. Vet Clin Pathol 31:140151. https://doi.org/10.1111/j.1939-165X.2002.tb00295.x

8. Hasegawa M, Fonteque J, Kohayagawa A, Boretti L (2002) Avaliação do perfil eletroforético das proteínas séricas em matrizes pesadas (Gallus Gallus Domesticus) da linhagem Avian Farm. Rev Bras Ciência Avícola 4:203-208. https://doi.org/10.1590/S1516-635X2002000300004

9. Hermier D (1997) Lipoprotein Metabolism and Fattening in Poultry. J Nutr 127:805S-808S. https://doi.org/10.1093/jn/127.5.805S

10. Hochleithner M (1994) Biochemistries. In: Ritchie, BW; Harrison, G.J.; Harrison LR (ed) Avian medicine: principles and application. Lake Worth: Wingers Publishing, pp 176-198

11. Hocking PM (1990) The relationships between dietary crude protein, body weight, and fertility in naturally mated broiler breeder males. Br Poult Sci 31:743-757. https://doi.org/10.1080/00071669008417305

12. Liberg $P$ (1977) Agarose gel electrophoretic fractionation of serum proteins in adult cattle. L A study of clinically healthy cows. Acta Vet Scand 18:40-53

13. Melillo A (2013) Applications of Serum Protein Electrophoresis in Exotic Pet Medicine. Vet Clin North Am Exot Anim Pract 16:211-225. https://doi.org/10.1016/j.cvex.2012.11.002

14. Meyer DL (1995) Medicina de Laboratório Veterinária: Interpretação e Diagnóstico. Roca, São Paulo

15. Penz AM, Jensen LS (1991) Influence of Protein Concentration, Amino Acid Supplementation, and Daily Time of Access to High- or Low-Protein Diets on Egg Weight and Components in Laying Hens. Poult Sci 70:2460-2466. https://doi.org/10.3382/ps.0702460

16. Proszkowiec-Weglarz M, Angel R (2013) Calcium and phosphorus metabolism in broilers: Effect of homeostatic mechanism on calcium and phosphorus digestibility. J Appl Poult Res 22:609-627. https://doi.org/10.3382/japr.2012-00743

17. Rajman M, Juráni M, Lamošová $D$, et al (2006) The effects of feed restriction on plasma biochemistry in growing meat type chickens (Gallus gallus). Comp Biochem Physiol Part A Mol Integr Physiol 145:363-371. https://doi.org/10.1016/j.cbpa.2006.07.004 
18. Ramírez F, Hobson KA, Wangensteen OS, et al (2010) A physiological marker for quantifying differential reproductive investment between the sexes in Yellow-legged gulls (Larus michahellis). J Exp Mar Bio Ecol 396:48-52. https://doi.org/10.1016/j.jembe.2010.09.012

19. Rath N, Balog J, Huff W, et al (1999) Comparative differences in the composition and biomechanical properties of tibiae of seven- and seventy-two-week-old male and female broiler breeder chickens. Poult Sci 78:1232-1239. https://doi.org/10.1093/ps/78.8.1232

20. Schmidt EMDS, Paulillo AC, Santin E, et al (2007) Hematological and serum chemistry values for the ring-necked pheasant (Phasianus colchicus): Variation with sex and age. Int J Poult Sci 6:137-139. https://doi.org/10.3923/ijps.2007.137.139

21. Silveira M, Freitas A de, Moraes C, et al (2014) Feeding management strategy for male broiler breeders and its effects on body weight, hatchability and fertility. Rev Bras Ciência Avícola 16:397402. https://doi.org/10.1590/1516-635X1604397-402

22. So HH, Jeon EO, Byun SH, Mo IP (2009) Early Diagnosis of Fatty Liver-Hemorrhagic Syndrome using Blood Biochemistry in Commercial Layers. Korean J Poult Sci 36:165-175.

https://doi.org/10.5536/KJPS.2009.36.2.165

23. Stout JD, Brinker DF, Driscoll CP, et al (2010) Serum Biochemistry Values, Plasma Mineral Levels, and Whole Blood Heavy Metal Measurements in Wild Northern Goshawks (Accipiter gentilis). J Zoo Wildl Med 41:649-655. https://doi.org/10.1638/2009-0258.1

24. Vin uela J, Ferrer M, Recio F, et al (1991) Age-related variations in plasma levels of alkaline phosphatase, calcium and inorganic phosphorus in chicks of two species of raptors. Comp Biochem Physiol Part A Physiol 99:49-54. https://doi.org/10.1016/0300-9629(91)90233-3

25. Walzern RL (1996) Lipoproteins and the laying hen: Form follows function. Avian Poult Biol Rev 7:31-64

\section{Supplementary Files}

This is a list of supplementary files associated with this preprint. Click to download.

- Supplementarytable.docx 